\title{
ISOTOPIC MIXED CRYSTAL EXCITON SPECTRA IN THE FAR INFRARED: NAPHTHALENE'
}

\author{
D.C. AHLGREN* and R. KOPELMAN \\ Department of Chemistry, University of Michigan, Ann Arbor, Michigan 48109, USA
}

Received 19 November 1979

\begin{abstract}
The far infrared vibrational exciton spectra of isotopic mixed crystals of naphthalene- $h_{8}$ and $d_{8}$ were studied. The two observed translational phonon modes were determined to fall into the amalgamated band limit while the lowest energy $B_{3 u}, A_{u}$ and $B_{1 u}$ vibrational exciton bands were found to be in the separated band limit. The lowest energy $B_{3 u}$ "butterfly" mode with its large $\left(15 \mathrm{~cm}^{-1}\right)$ exciton splitting was found to agree well with CPA calculations of mixed crystal spectra. A peak at $185 \mathrm{~cm}^{-1}$ was also assigned as a peak in the vibrational exciton density-of-states of the $B_{3 u}$ mode.
\end{abstract}

\section{Introduction}

Isotopic mixed crystal studies have long been used for the elucidation of vibrational exciton and phonon interactions in molecular solids [1-4]. Concentrated binary isotopic mixed crystals provide a nearly ideal system for studying the exciton band structure of molecular crystals. Although the entire band structure and direct trap absorptions are not normally observed, the extent of the exciton band can usually be monitored, as in the case of naphthalene, by the two Davydov components which usually occur close to the extremes of the band [5-7]. Because $k$ is not a good quantum number in the mixed crystal, statistical methods are used to calctiate an average hamiltonian for the mixed crystal and Green's function methods have been used to calculate band structures and optical spectra of concentrated binary mixed crystals $[5,6]$.

Exciton bands of isotopic mixed crystals can be classified according to two limits [7]. In the separated band limit ("two mode case") there exists a band gap between guest and host bands. This case occurs when the trap depth (isotope shift, energy denominator) $\Delta$ is large compared to the pure guest

t Supported by NIH Grant NSO8116-10A1.

* Present address: IBM - East Fishkill Facility, Department 63A, Hopewell Junction, New York 12533, USA. or host bandwidth $B$. If, on the other hand, the trap depth is small compared to the exciton bandwidth, we find that a single mixed-crystal exciton band exists for all guest and host concentrations. This is characteristic of the amalgamation limit ("single mode case"). In the following discussion we shall demonstrate that the infrared active translational phonon modes of naphthalene are characteristic examples of an amalgamated exciton band, and the $B_{3 u}, A_{u}$ and $B_{1 u}$ vibrational exciton bands at $185 \mathrm{~cm}^{-1}, 210 \mathrm{~cm}^{-1}$ and $362 \mathrm{~cm}^{-1}$, respectively, fall into the separated band limit.

\section{Experimental}

Matheson, Coleman and Bell recrystallized naphthalene was used for the naphthalene- $h_{8}$ samples, and perdeuteronaphthalene with an isotopic purity of 99.52 atom $\% \mathrm{D}$ purchased from Wilmad Glass Co., Inc. was used for the naphthalene- $d_{8}$ spectra. Samples of about $1 \mathrm{~mm}$ thickness were grown directly on aluminum sampie holders with an optical window diameter of $5 / 8$ inch by allowing liquid naphthalene to cool slowly over a 4 hour period. This allowed a poly-crystalline. sample to be formed so that all polarizations could be observed. Neat naphthalene- $h_{8}$ and $d_{8}$ crystals as well as isotopic mixed crystals with $h_{8}: d_{8}$ ratios of 
(a)

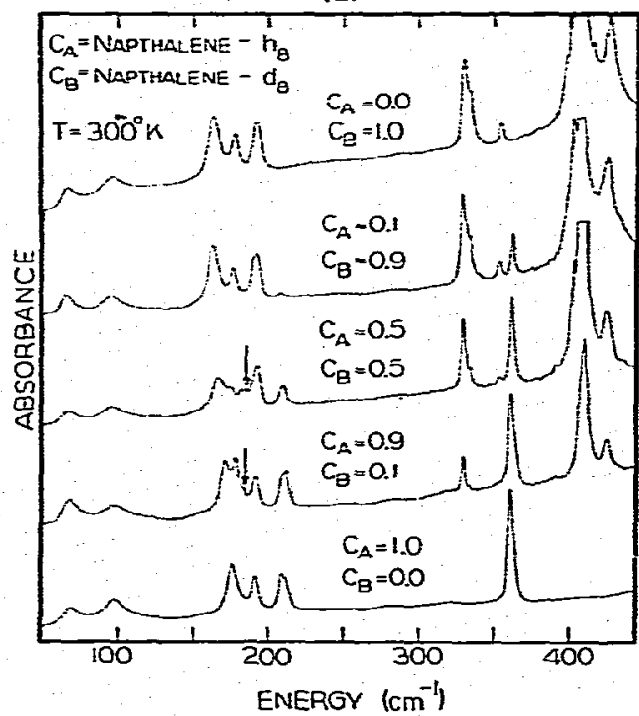

Fig. 1. These isotopic mixed naphthalene crystal spectra with $C_{10} D_{8}$ mole fractions $\left(C_{B}\right)$ in the 0 to 1 range demonstrate the difference in behavior between the amalgamation limit $\left(a_{u}\right.$ and $b_{u}$ phonon peaks, below $100 \mathrm{~cm}^{-1}$ ) in which a monotonic frequency shift is apparent, and the separated band limit (internal modes, above $150 \mathrm{~cm}^{-\mathrm{t}}$ ) which exhibit a more complex concentration dependence. Fig (a) spectra were taken at $300 \mathrm{~K}$, while the fig. (b) spectra were taken at $78 \mathrm{~K}$. The arrow in the $300 \mathrm{~K}$ spectra marks the defect/impurity induced maximum of the vibraticnal exciton density-of-states at $185 \mathrm{~cm}^{-1}$.

$10: 90,50: 50$ and $90: 10$ were grown, and all spectra were taken on a Digilab FTS-16 Fourier transform spectrometer in double precision with a resolution of $1 \mathrm{~cm}^{-1}$. The low temperature spectra were taken using a cold finger sample holder made of OFHC copper which was filled with acetone/dry-ice or liquid nitrogen. The cold finger was mounted in a stainless steel evacuated jacket with wedged polyethylene windows to eliminate interference fringing effects. Thermal contact was made between the cold finger and the aluminum sample holder with indium gaskets, and the temperature was checked using a thermocouple mounted between the indium gasket and the crystal.

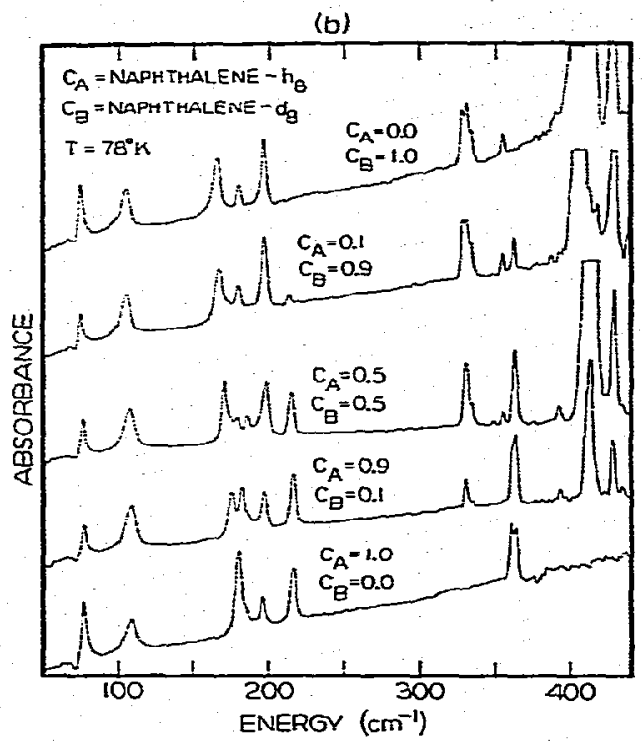

\section{Results and discussion}

\subsection{Translational phonons and the amalgamation limit}

The low energy infrared region of several naphthalene isotopic mixed crystals is shown at $300 \mathrm{~K}$ in fig. la. The peaks at 69 and $98 \mathrm{~cm}^{-1}$ are the $q=0 a_{u}$ and $b_{u}$ phonon peaks, respectively. No third $q=0$ infrared active mode was found although we did not look below $50 \mathrm{~cm}^{-1}$. This third $q=0$ phonon was reported at $55 \mathrm{~cm}^{-1}$ by Harada and Shimanouchi [8], but Hadni et al. [9], using polarization data, have concluded that two phonon $q=0$ modes lie at $96 \mathrm{~cm}^{-1}$, one with electric dipole component in the $a c$ plane and one along the monoclinic $b$ axis. The 69 and $98 \mathrm{~cm}^{-1}$ peaks are shifted to 67 and $96 \mathrm{~cm}^{-1}$ in neat perdeuteronaphthalene. A similar 2 to $3 \mathrm{~cm}^{-1}$ isotope sinift can be seen in the spectra taken at $78 \mathrm{~K}$ (fig. Ib). Althugh the two observed $q=0$ peaks belong to different phonon bands and do not define the extent of these bands, the phonon bandwidth is considerably larger (typically several tens of $\mathrm{cm}^{-1}$ ) than this $2 \mathrm{~cm}^{-1}$ "trap depth". As a result, we would expect the phonon band to fall into the amaigamation limit. 


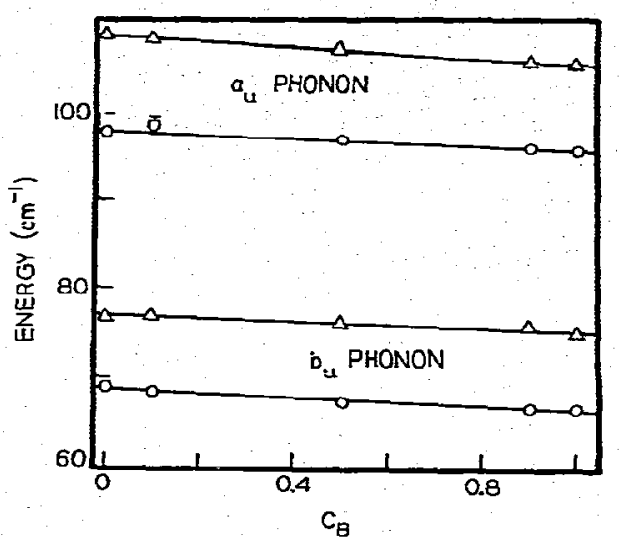

Fig. 2. A monotonic shift of naphthalene $a_{u}$ and $b_{u}$ phonon bands as a function of $C_{10} D_{8}$ concentration $\left(C_{B}\right)$ can be seen. The circles are room temperature $(300 \mathrm{~K})$ data while the.triangular points were taken at $78 \mathrm{~K}$. This behavior is similar to results for acetylene [13] and durene [11].

Fig. 2 demonstrates the expected monotonic shift of the frequency of these phonon peaks upon isotopic mixing. indicating a single amalgamated phonon band rather than separate guest and host bands. This behavior has been observed and discussed for the Raman active librational phonon modes of naphthalene [10], and in the case of durene [11], benzene [12] and acetylene [13].

\subsection{Vibrational excitons and the separated band limit}

The difference in intramolecular vibrational energy caused by a change in reduced mass upon complete deuteration of the naphthalene molecule is generally large compared to the intermolecular interactions (environmental effects) which give rise to exciton bandwidth. As a result there is normally a large energy separation between the perproto and perdeuteronaphthalene exciton bands for a particular vibrational modc, and vibrational exciton bands of isotopic mixed crystals fall into the separated band limit. The $B_{1 u}$ vibration which occurs at $362 \mathrm{~cm}^{1}$ in naphthalene- $h_{8}$ and $329 \mathrm{~cm}{ }^{1}$ in naphthalene- $d_{8}$ at $300 \mathrm{~K}$ is typical of this behavior. The mixed crystal spectra in fig. la show the effect of isotopic dilution on the $B_{1 u}$ vibration. In the three intermediate concentrations there is a well defined $30 \mathrm{~cm}^{-1}$ band gap separating the " $h_{8}$ " and " $d_{8}$ " $B_{1 u}$ bands. We observe that both intensity and bandwidth for the naphthalene- $h_{8}$ and $d_{8}$ bands decrease upon isotopic dilution. The intensity effect is a consequence of Beer's law. The contraction of bandwidth (splitting), illustrated in table 1 , is a result of a decrease in the number of interactions among naphthalene- $h_{8}$ molecules in the $-h_{8}$ band (naphthalene- $d_{8}$ molecules in the $-d_{8}$ band) causing a merging of the (now unresolved) Davydov components.

The decrease in exciton bandwidth upon isotopic dilution in mixed crystals can be a useful tool in the assignment of Davydov components in pure crystals. Exciton splittings are normally identified through polarization studies but often incorrect conclusions are drawn from ambiguous data. The heavily doped isotopic mixed crystal method for determination of exciton splittings has proven useful in the assignment of the Raman active vibrational spectrum of naphthalene [14]. This method involves monitoring proposed Davydov components of a single exciton band in the isotopic mixed crystal. In the separated band limit, the exciton splitting should decrease upon isotopic dilution because of the contraction of exciton bandwidth discussed earlier. In the limit of a dilute isotopic mixed crystal, we sce only a single monomer peak. Two peaks belonging to different exciton bands (i.e., modes of distinct molecular parentage) should show no merging in mixed crystal spectra. On this basis, we can assign peaks at 329 , $334,354,407$ and $427 \mathrm{~cm}^{-\frac{1}{2}}$ in perdeuteronaphthalene (shown in fig. la) to separate vibrational exciton bands. The 334 and $354 \mathrm{~cm}^{-1}$ bands are probably due to partially deuterated isotopic impurities. Structure seen on the bands at 407 and $427 \mathrm{~cm}^{-1}$ is due to an increased noise level above $400 \mathrm{~cm}^{-1}$ caused by decreased beam-splitter efficiency.

Table 1

$B_{1 u}$ vibrational exciton bandwidths $\left(\mathrm{cm}^{-1}\right)$

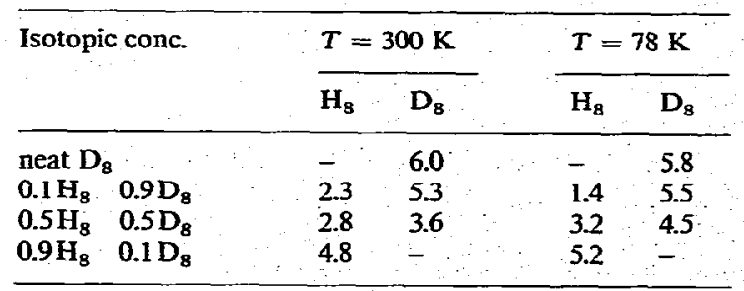


In the $200 \mathrm{~cm}^{-1}$ region, shown in fig. la at $300 \mathrm{~K}$, we see a more complicated behavior. It can be seen from the schematic representation of this region in fig. 3a that the two lower energy peaks in the neat crystal belong to a single exciton band with a bandwidth of about $15 \mathrm{~cm}^{-1}$. The higher energy peak belongs to a vibrational exciton with i considerably smaller splitting - about $3 \mathrm{~cm}^{-1}$ for naphthalene- $h_{\mathrm{g}}$ at $300 \mathrm{~K}$ and less than $1 \mathrm{~cm}^{-1}$ for naphthalene- $d_{\mathrm{s}}$. The $15 \mathrm{~cm}^{-1}$ splitting in the lower naphthalene- $h_{\mathrm{g}}$ band decreases to $8 \mathrm{~cm}^{-1}$ in the $50 \%$ crystal and we see only a single peak at $185 \mathrm{~cm}^{-1}$ in the dilute $(10 \%) h_{8}$ in $d_{8}$ crystal A similar behavior is seen in the lower energy band in naphthalene- $d_{\mathrm{s}}$ which is split by $14 \mathrm{~cm}^{-1}$ in the neat $d_{\mathrm{s}}$ crystal and decreases to $1.5 \mathrm{~cm}^{-1}$ in the $90 \%$ $h_{8}$ in $d_{8}$. The higher energy vibration at $211 \mathrm{~cm}^{-1}$ in $\mathrm{C}_{10} \mathrm{H}_{8}$ also exhibits this decrease in exciton splitting upon isotopic dilution. No splitting of this mode is resolved in $\mathrm{C}_{10} \mathrm{D}_{\mathrm{g}}$. Spectra of these isotopic mixed crystals taken at $78 \mathrm{~K}$, shown in fig. $3 \mathrm{~b}$ schematically, exhibit similar effects of isotopic mixing with better resolution. We have thus assigned the peaks in the $200 \mathrm{~cm}^{-1}$ region of naphthalene to two separate vibrational exciton bands, one with a large Davydov splitting of about $15 \mathrm{~cm}^{-1}$ and one with a small $2-3 \mathrm{~cm}^{-1}$ splitting. This is in agreement with Bree and Kydd [15] who have assigned the lower vibration as $B_{3 u}$ and the higher one as $A_{u}$ on the basis of polarization data, as well as with Duckett et al. [16]. whose gas-phase assignments were made on the basis of band contours, and with the force field calculations of Pietila and Stenman [17].

\subsection{The largest and lowest energy vibrational exciton band}

The $B_{3 v}$ vibrational exciton band with its Davydov components at 177 and $192 \mathrm{~cm}^{-1}$ in $\mathrm{C}_{10} \mathrm{H}_{8}$ and 163 and $177 \mathrm{~cm}^{-1}$ in $\mathrm{C}_{10} \mathrm{D}_{8}$ (at $300 \mathrm{~K}$ ) is the lowest energy intramolecular vibration in the naphthalene crystal. This band corresponds to the out-of-plane butterny motion of the two rings in the naphthalene molecule [18]. A $15 \mathrm{~cm}^{-1}$ exciton splitting makes this also the widest vibrational exciton band in both the infrared and the Raman spectrum $[14,19]$. Interactions for this motion may be expected to be large between nearest neighbor
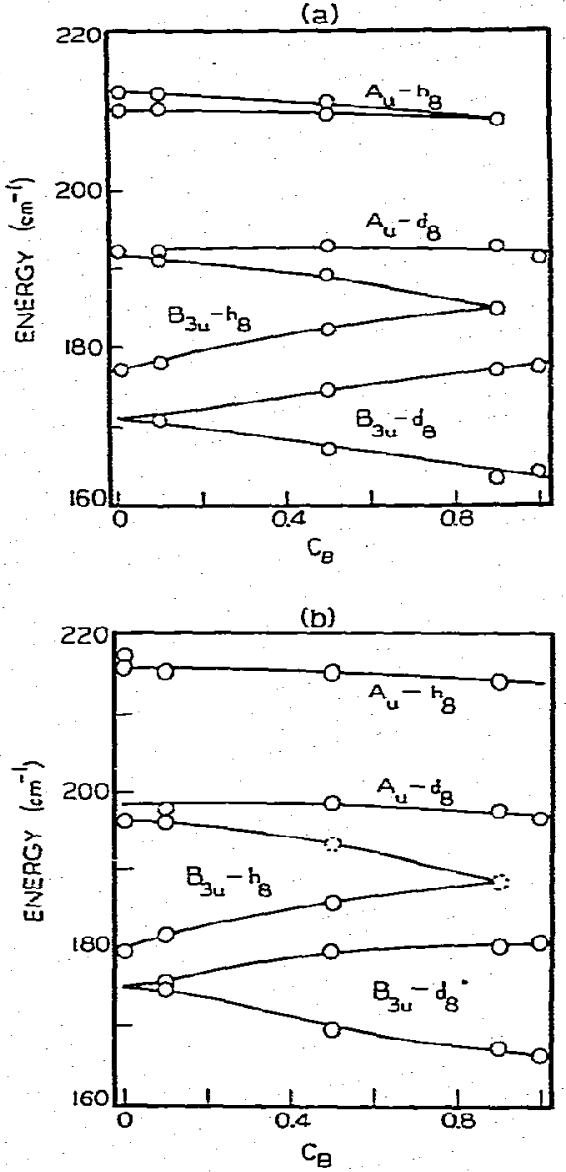

Fig. 3. This is a schematic representation of the $A_{u}$ and $B_{34}$ absorption peak locations as a function of $C_{10} D_{8}$ concentration $\left(C_{B}\right)$. The $B_{3 u}$ band shows a very large $\left(15.0 \mathrm{~cm}^{-1}\right)$ splitting in the neat $\mathrm{C}_{10} \mathrm{H}_{8}$ crystal $\left(14.2 \mathrm{~cm}^{-1}\right.$. in neat $\mathrm{C}_{10} \mathrm{D}_{8}$ ) at $300 \mathrm{~K}$ (fig. (a)). The decrease in exciton bandwidth (as monitored by the two "Davydov components") with isotopic dilution is typical of the separated band limit. At $78 \mathrm{~K}$ (fig. (b)), the Davydov splitting in the $B_{34}$ mode is about $16.7 \mathrm{~cm}^{-1}$ in neat $\mathrm{C}_{10} \mathrm{H}_{8}$ and $14.4 \mathrm{~cm}^{-1}$ in neat $\mathrm{C}_{10} \mathrm{D}_{8}$.

interchange equivalent molecules (center-to-center distance of $5.11 \AA$ ) in the $a b$ plane and small for all other interchange and translationally equivalent pairs. This nearest neighbor interaction results, presumably, in the large exciton splitting of this 
out-of-plane mode. However, an asymmetry is revealed in the incoherent inelastic neutron curve and agreement with simple theoretical models was found to be poor by Bokhenkov et al. [20]. These authors also considered the "Dayydov splitting" $\left(17 \mathrm{~cm}^{-1}\right)$ of this mode to be a tentative assignment.

The observed mixed crystal $B_{3 u}$ exciton spectra are in very good qualitative agreement with the coherent potential approximation calculations performed by Hoshen and Jortner [6] and Hong and Robinson [21] for cases in which the trap depth is about the same magnitude as the exciton bandwidth. A comparison of our results (shown with the $A_{u}$ exciton band subtracted out) with calculated optical spectra of Hong and Robinson [21] can be seen in fig. 4 . The $B_{3 u}$ exciton band is therefore demonstrated to fall (barely) into the separated band limit with a trap depth of $13 \mathrm{~cm}^{-1}$ and a bandwidth of $15 \mathrm{~cm}^{-1}$.

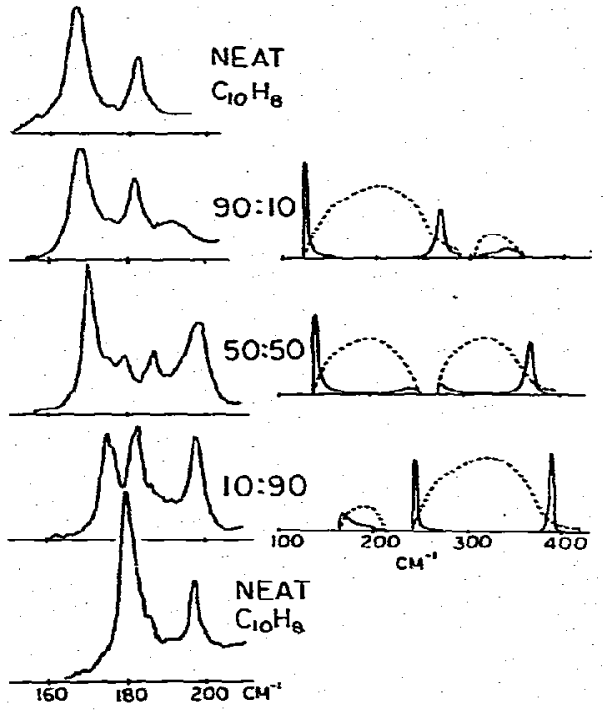

Fig. 4. Experimental data for the $B_{3 u}$ vibrational exciton band (left) at $78 \mathrm{~K}$ with the $A_{u}$ peak removed, are compared to a calculation of the naphthalene exciton bands (dashed lines) with $\Delta \approx B$ performed by Hong and Robinson [21] (right). The calculated optical spectrum (solid lines) is in good agreement with spectral peak intensities and positions. Experimentally, $\Delta=13 \mathrm{~cm}^{-1}$ and $B=15 \mathrm{~cm}^{-1}$ while $\Delta=115 \mathrm{~cm}^{-1}$ and $B=175 \mathrm{~cm}^{-1}$ for the calculated exciton bands. Rigorously, the energies should have been scaled by the $\Delta$ 's and the bands have identical $B / \Lambda$.
In $10 \% \mathrm{C}_{10} \mathrm{H}_{8}$ in $\mathrm{C}_{10} \mathrm{D}_{8}$ at $300 \mathrm{~K}$ we see a single peak at $185 \mathrm{~cm}^{-1}$ due to $\mathrm{C}_{10} \mathrm{H}_{8}$ monomers and other small clusters. This peak is roughly at the center of the $\mathrm{B}_{34} \mathrm{C}_{10} \mathrm{H}_{8}$ exciton band. In fig. la we see a feature at $185 \mathrm{~cm}^{-1}$ occurring in the $50 \%$ and $90 \% \mathrm{C}_{10} \mathrm{H}_{8}$ in $\mathrm{C}_{10} \mathrm{D}_{8}$ and very weakly in the neat $\mathrm{C}_{10} \mathrm{H}_{8}$ crystal. We have tentatively attributed this $185 \mathrm{~cm}^{-1}$ feature to a peak in the $B_{3 u}$ vibrational exciton density-of-states, a transition which becomes weakly allowed as the $\Delta k=0$ selection rule breaks down in isotopic mixed crystals (and to a certain extent in the neat crystal with natural defects and/or impurities present). This effect has been noted also in the infrared spectrum of solid benzene [22]. Similar peaks at the center of the $B_{3 u}$ exciton bands in both naphthalene- $h_{\mathrm{B}}$ and $d_{8}$ also seem to be present in the spectra taken at $78 \mathrm{~K}$, however, no assignment has been made due to a higher noise level in these spectra.

\section{References}

[1] G.L. Hiebert and D.F. Hornig, J. Chem. Phys. 20 (1952) 918.

[2] E.R. Bernstein, S.D. Colson, R. Kopelman and G.W. Robinson, J. Chem. Phys. 48 (1968) 5596.

[3] F.P. Chen and P.N. Prasad, J. Chem. Phys. 66 (1977) 4341; Chem. Phys. Letters 47 (1977) 341.

[4] R.G. Whitfield and G.E. Leroi, J. Chem. Phys. 68 (I978) $2151 ; 4384$.

[5] H.-K. Hong and R. Kopelman, J. Chem Phys. 55 (1971) 724.

[6] H. Hoshen and J. Jortner, J. Chem. Phys. 56 (1972) 5550.

[7] R. Kopelman, in: Excited states, Vol. 2, ed. E.C. Lim (Academic Press, New York, 1975).

[8] I. Harada and T.H. Shimanouchi, J. Chem. Phys. 44 (1966) 2016.

[9] A. Hadni, B. Wyncke, G. Morlot and X. Gerbaux, J. Chem. Phys. 51 (1969) 3514:

[10] P.N. Prasad and R. Kopelman, J. Chem. Phys. 57 (1972) 863.

[11] P.N. Prasad and R. Kopelman, J. Chem. Phys. 58 (1973) 5031 .

[12] H.-K. Hong and R. Kopelman, J. Chem. Phys. 58 (1973) $384,2557$.

[13] C.S. Blackwcll, R. Kopelman and G. Leroì, in: Molecular Spectroscopy of Dense Phases. Proceedings of the 12th European Congress on Molecular Spectroscopy, Strasbourg, France, July 1-4, 1975, eds. M. Grossmann, S.G. Elkomos and J. Ringeissen (Elsevier, Amsterdam, 1976). 
[14] P.N. Prasad and R. Kopelman, I. Chem. Phys. 57 (1972) 856

[15] A. Bree and R.A. Kydd, Spectrochim. Acta 26A (1971) 1791.

[16」 J.A. Duckett, T.L. Smithson and H. Weiser, J. Mol. Spectry. 44 (1978) 97.

[17] L.O. Pietila and F. Stenman, Commentat. PhysMath. 48 (1978) 145.

[18] E.R. Lippincott and E.J. O'Reilly, J. Chem. Phys. 23 (1955) 283.
[19] G.S. Pawley and S.J. Cyvin, J. Chem. Phys. 52 (1970) 4073.

[20] E.L. Bokhenkov, I. Natkaniec and E.F. Sheka, Soviet Phys. JETP 43 (1976) 536; Phys. Stat. Sol. 75 (1976) 105.

[21] H.-K. Hong and G.W. Robinson, J. Chem. Phys. 52 (1970) 825.

[22] J.C. Laufer and R. Kopelman, J. Chem. Phys. 57 (1972) 3202 